\title{
Translational realistic expectations of chronic cerebral hypoxemia in rat model after bilateral commom carotid artery ligation. Neurocognitive aspects ${ }^{1}$
}

\author{
Maria Cecília Santos Cavalcanti Melo ${ }^{\mathrm{I}}$, Diego Gadelha" ${ }^{\mathrm{II}}$, Guilherme Veras Mascena ${ }^{\mathrm{III}}$, Thárcia Kiara Beserra Oliveira ${ }^{\mathrm{IV}}$, \\ Carlos Teixeira Brandt ${ }^{\mathrm{v}}$
}

IFellow PhD degree, Postgraduate Program in Surgery, Federal University of Pernambuco (UFPE). Assistant Professor, Department of Ophthalmology, Campina Grande Faculty of Medicine (FCM), Campina Grande-PB, Brazil. Acquisition and interpretation of data, manuscript writing.

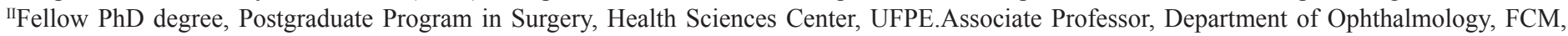
Campina Grande-PB, Brazil. Manuscript writing, critical revision.

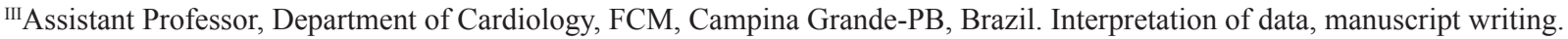

${ }^{\text {IV }}$ Veterinarian, Fellow Master degree in Agricultural Engineering - UFCG - Paraíba , Federal University of Campina Grande (UFCG), Campina Grande-PB, Brazil. Acquisition and interpretation of data

vPhD, Head Professor, Scientific Methodology and Pediatric Surgery, UFPE, Recife-PE, Brazil. Coordinator of Campina Grande Faculty of Medicine (FCM), Campina Grande-PB, Brazil. Scientific and intellectual content of the study, interpretation of data and final critical revision.

\begin{abstract}
PURPOSE: To evaluate the effects of chronic cerebral hypoxia on memory of rats submitted to bilateral common carotid artery ligation (BCCL).

METHODS: Every each week, for 16 weeks, 31 rats were tested for memory using a water and land mazes and compared with 30 normal rats (control group A). The variables were expressed by their mean and standard error of the mean (SEM). p $<0.05$ was used for rejecting the null hypothesis. The study was approved by the Ethics Committee for animal investigation.

RESULTS: There was a significant increase in the latency time, in the survival water and land mazes, after four weeks (study group B) follow-up. However, without any medication or therapeutically induced measures, after 16 weeks (study group C) follow-up the latency mean times tend to be similar to control group (A) in the neurocognitive tests.

CONCLUSIONS: Neurocognitive deficits after 16 weeks post-operative follow-up of rats that underwent bilateral common carotid artery ligation is a natural adaptive phenomenon. Thus, is not realistic to allow translational information from this animal model for therapeutically approaches aiming at to prevent, or to improve brain damage in human beings suffering from chronic deprivation of adequate blood supply.
\end{abstract}

Key words: Hypoxia-Ischemia, Brain. Carotid Arteries. Memory Disorders. Rats. 


\section{Introduction}

Since early 1960s, although the mortality rate in Sprague- Dawley rats was very high when both carotid arteries were permanent occluded $^{1}$, it was realized that in Wistar rats, consistent ischemic damage of the brain was very difficult to maintain consistentely ${ }^{2}$.

Permanent occlusion of both common carotid arteries in the rat or bilateral common carotid artery ligation (BCCL) has been used as an experimental model for chronic cerebral hypoperfusion ${ }^{3}$. After BCCL, cerebral blood flow (CBF) immediately declines to $30-60 \%$ of its control value, but recovers to approximately $\sim 63 \%$ and $\sim 90 \%$ of control at four- and eightweeks, respectively ${ }^{4,5}$.

Acute BCCL in rats leads to increase in intracellular calcium $^{6}$ to activate ARA-selective cPLA2 and release ARA and increase ARA recycling in phospholipid ${ }^{7}$. Activation of SPLA2 by calcium $^{8}$ also may release ARA and ultimately to produce $\mathrm{PGE}^{9}$ to mediate compensatory vasodilatation. Nitric oxide, free radicals and neurogenic factors during ischemia also can produce vasodilatation $^{10,11}$. It is likely that some recovery occurred in the present study even at $24 \mathrm{~h}$, associated with the sPLA2 activation, helping to normalize the significant disturbances in UFA and ARACoA concentrations evident at $6 \mathrm{~h}$. However, auto regulation remains abnormal and the brain is more vulnerable to additional insults such as hypotension, hypoxia and further ischemia following $\mathrm{BCCL}^{12}$.

Several therapeutically approaches have been followed to prevent or to improve the neurocognitive deficit produced by $\mathrm{BCCL}^{13-}$ ${ }^{24}$. Among them: nitric oxide carrier such as S-nitrosoglutathione (GSNO) $)^{13}$; several natural substances like ligustilide (LIG), a main lipophilic component of Danggui (Chinese Angelica root, Radix Angelica sinensis) $)^{14}$; Bushen Jiannao Recipe (BJR) ${ }^{15}$; effective component group of Chinese herbal medicine ${ }^{16}$; or biochemical substances like DL-3-n-butylphthalide (DL-NBP) $)^{17}$, ferulic acid ${ }^{18}$, 2D-DIGE combined with MALDI-TOF MS ${ }^{19}$, Insulin-like growth factor-1 (IGF-1) $)^{20}$; or yet cognitive and physical stimulation, named Environmental Enrichment, consisted of one-hour sessions run 3 times per week during 12 weeks $^{21}$; electro acupuncture combined with compound Salviae Miltiorrhizae tablet ${ }^{22}$; a mixed compound of ginkgo biloba extract and cilostazol ${ }^{23}$, or administration of Pistacia lentiscus L. essential oil ${ }^{24}$, just to list the investigations produced in the year 2012. However, none of these studies or the previous ones follow the clinical course of this condition long enough to evaluate the natural history of the so called neurocognitive chronic effect of BCCL without doing any kind of intervention.
The purpose of this investigation was to weekly evaluate the neurocognitive status of the rats that underwent BCCL, without any intervention on the natural history with the minimum followup of 16 weeks.

\section{Methods}

The research project was approved by the Research Ethics Committee of FCM-Campina Grande-PB, Brazil.

Adult Wistar rats (Rattus norvegicus) from the animal colony were used for this study. They were housed in polypropylene cages, four animals per cage, under standard light/dark conditions (lights on 7:00, off 19:00) with food pellets and water ad libitum.

Chronic cerebral hypo perfusion was induced by BCCL ${ }^{1-22}$, in 31 rats. The animals were anesthetized with ketamine hydrochloride $(50 \mathrm{mg} / \mathrm{kg}$ - ip) and xilasine (10 mg/kg -ip). The common carotid arteries were exposed via a ventral midline incision, carefully separated from their sheaths and vagus nerves, and permanently doubly tied with $5 / 0$ silk suture approximately 8 to $10 \mathrm{~mm}$ below the origin of the external carotid artery.

Every each week, for at least sixteen weeks, the rats were tested for memory and learning, using a survival water maze task (SWMT). The apparatus consisted of a rectangular water tank measuring $120 \mathrm{~cm}$ in length by $80 \mathrm{~cm}$ wide and $60 \mathrm{~cm}$ in height. To make the water opaque, $1 \mathrm{~kg}$ of powdered milk was added, and water temperature was kept at $23 \pm 1^{\circ} \mathrm{C}$. A translucent acrylic platform (10 $\mathrm{cm}$ in diameter) was located in the one end (north) of the maze during training. The top of the platform was approximately $1.5 \mathrm{~cm}$ below the surface of water (Figure 1).

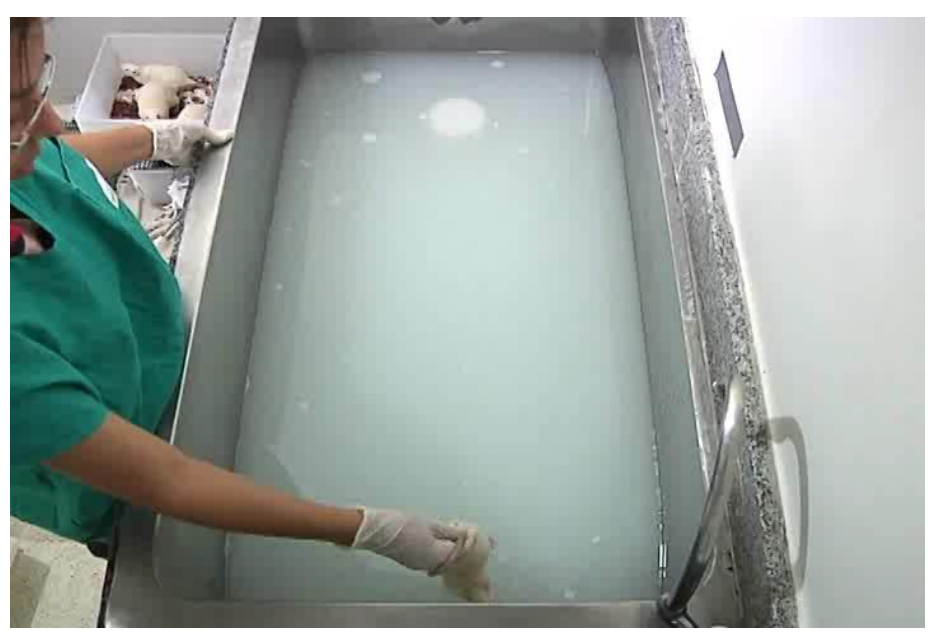

FIGURE 1 - Rectangular survival water maze. Rat starting position for the survival learning memory. Submerse circular platform close to the south end of the maze in opposition of rat initial water mark. 
Spatial training of the platform in the water maze was performed for five consecutive days. Each rat received two trials per day for five days with the inter trial interval of twelve hours.

The starting position (south end) for each trial was randomly chosen and counterbalanced across all experimental groups.

The rats were gently placed into the water, facing the middle position of the maze south side wall. Swimming paths of the rats were monitored by a video camera. For each training trial, the latency to escape onto the platform and the path length were recorded. All data were fed into a computer program for posterior independent analysis.

The rats were given a maximum of $60 \mathrm{~s}$ to find the platform. If they failed to find it within $60 \mathrm{~s}$, the training was terminated and a maximum score of $60 \mathrm{~s}$ was assigned. The rats were then guided to the platform by hand, and it was allowed to stay on it for 10 s before removed from the water.

The physical findings, the way of swimming, balance instability and weakness of the limb muscles were also recorded and analyzed. Both the training process and the sixth day evaluations were performed in a randomized fashion in order to eliminate any bias in the analysis.

Similarly, the rats were tested for visual and olfactory functions in a land maze (Figure 2).

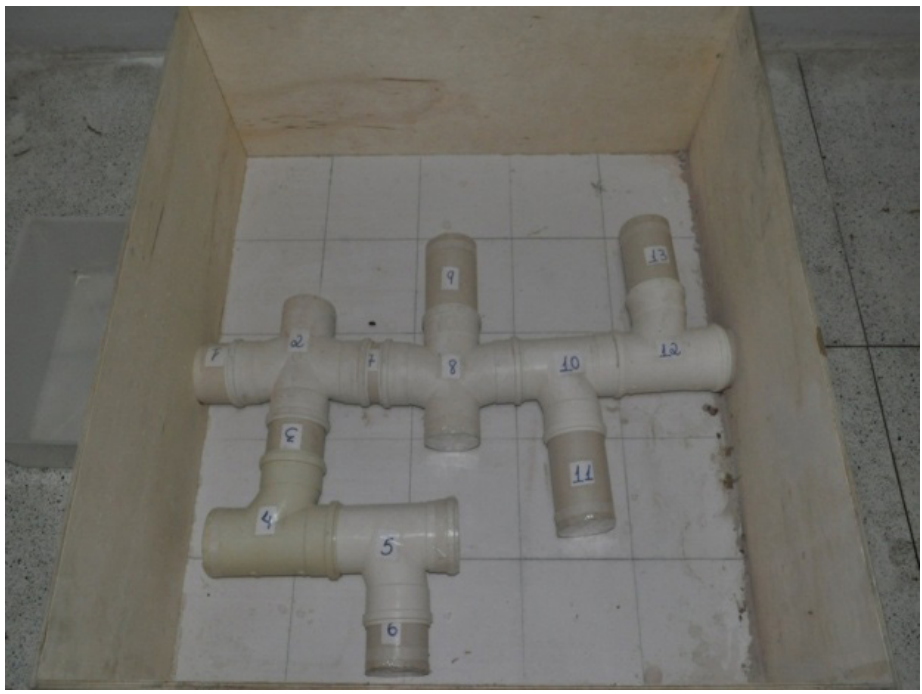

FIGURE 2 - Land maze for testing visual and olfactory functions.

The animals were maintained in starving condition on individual cages for 24 hours.

Each rat was placed in the entrance of the land maze and the time was recorded until the rat found the exit of the maze where its original cage with food and water were placed. The environment was in the shadows just with a weak monochromatic blue light. For each week the latency times were recorded and analyzed after four weeks (study group B) and 16 weeks (study group C). In this meantime the animals were housed in polypropylene cages, four animals per cage, under standard light/dark conditions (lights on 7:00, off 19:00) with food pellets and water ad libitum.

Thirty normal rats without any disease (control group A) were caged in the same environment and way of life; including food and water,

The quantitative variables were expressed by their mean and standard error of the mean (SEM). $\mathrm{p}<0.05$ was used for rejecting the null hypothesis.

\section{Results}

The mortality rate for BCCL was $38.7 \%$

There was a significant increase in the latency time, in the survival water maze, after four weeks (study group B) followup. However, without any medication or therapeutically induced measures, after 16 weeks (study group C) follow-up the latency mean time tends to be similar to control group (A) (Figures 3 and 4).

O ne-w a y NOVA d a ta

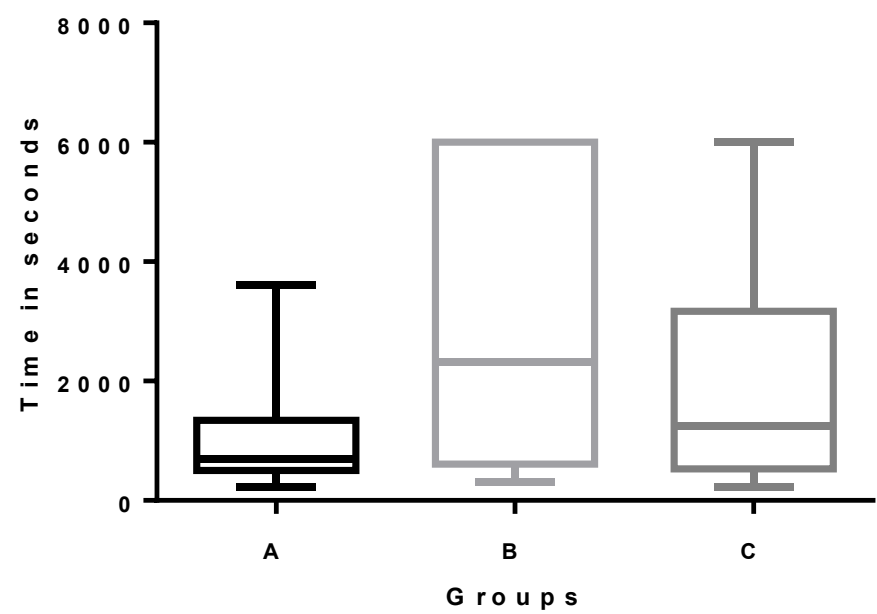

FIGURE 3 - Box plot of the medians of survival water maze times from the control group (A) and studied groups at four weeks follow-up (study group B) and at more than 16 weeks follow-up (study group $\mathbf{C}$ ) $-\mathrm{p}=0.0009$. 
$95 \%$ C onfidence Intervals (Tukey)

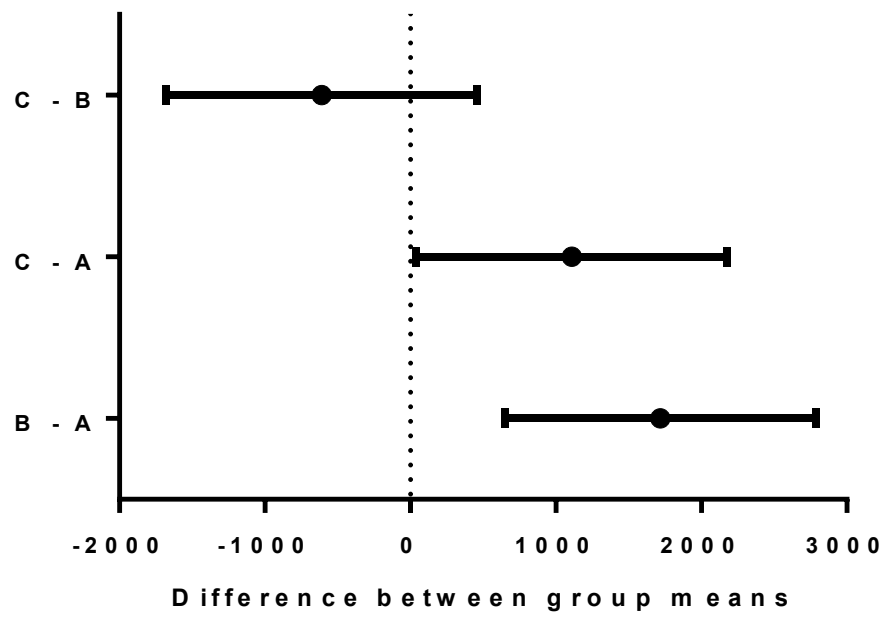

FIGURE 4 - Tukey post-test of survival water maze for comparing the control group (A) and studied groups at four weeks follow-up (study group B) and at more than 16 weeks follow-up (study group C). No difference comparing latency times from $\mathrm{C}$ and $\mathrm{B}-\mathrm{p}=0.3680$; small, but significant difference, comparing the mean latency time from groups $\mathrm{C}$ and $\mathrm{A}-\mathrm{p}=0.0403$; and significant difference comparing study group $\mathrm{B}$ and control group A $-\mathrm{p}=0.0007$.

There was a significant increase in the latency time, in the survival land maze, after four weeks (study group B) follow-up. However, without any medication or therapeutically induced measures, after 16 weeks (study group C) follow-up the latency mean time was even significant smaller than the control group (A) (Figures 5 and 6).

As a whole, the latency mean times of water survival maze increased at four weeks follow-up; but, they tend to be similar to normal (control) rats after 16 weeks follow-up, without any treatment measure. Similarly, as regard to land maze times, they increase at four

\section{O n e -w a y N O V A d a ta}

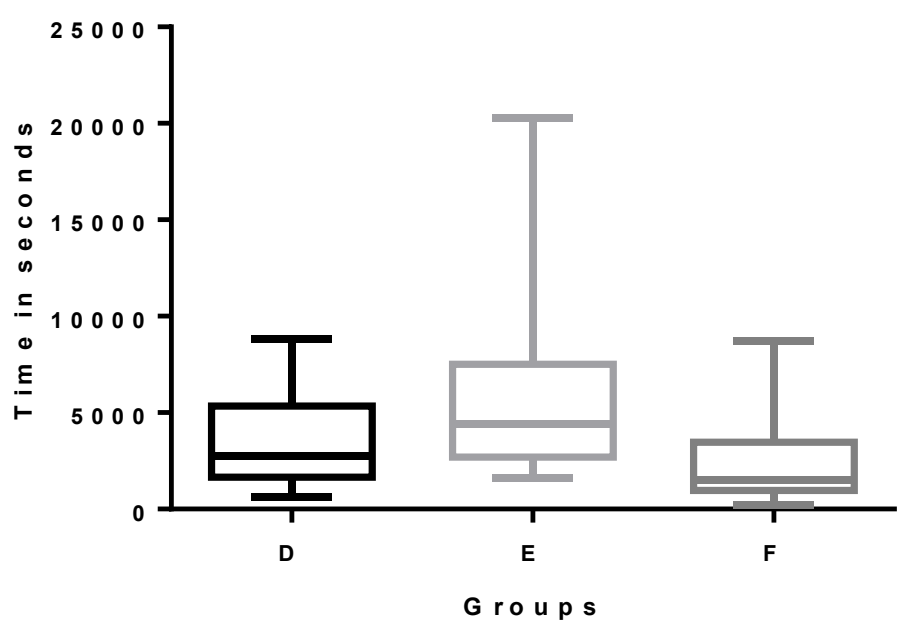

FIGURE 5 - Box plot of the medians of survival land maze times from the control group ( $\mathbf{A}$ - represented by $\mathbf{D})$ and studied groups at four weeks follow-up (study group $\mathbf{B}$ - represented by $\mathbf{E}$ ) and at more than 16 weeks follow-up (study group $\mathbf{C}-$ represented by $\mathbf{F}$ ) $-\mathrm{p}=0.0003$.
$95 \%$ C onfidence Intervals (T ukey)

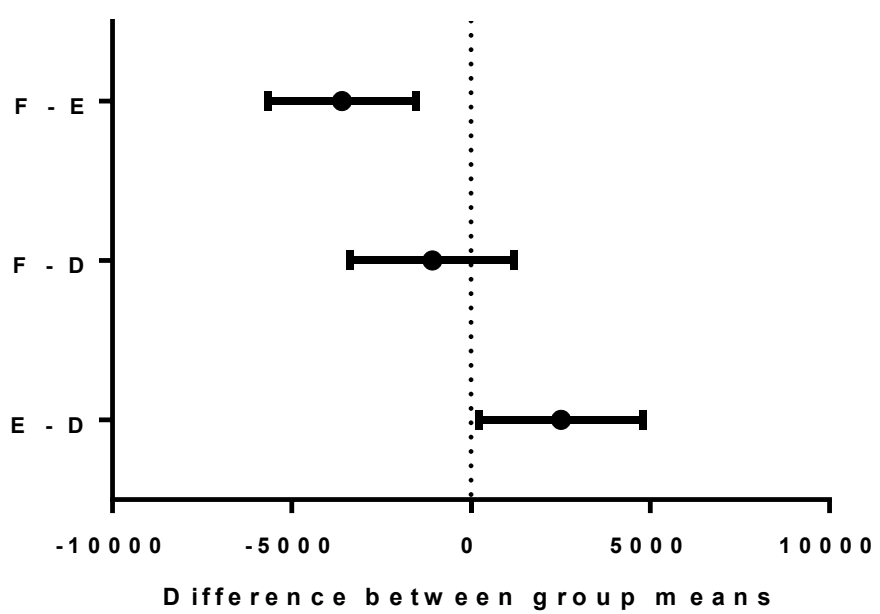

FIGURE 6 - Tukey post-test of survival land maze for comparing the control group (A) and studied groups at four weeks follow-up (study group B) and at more than 16 weeks follow-up (study group C). Significant increase in the mean land maze latency time comparing study group B (E) and control group $A(\mathbf{D})-p=0.0277$. No difference between the land maze latency times of control group A (D) and study group C (F) - $\mathrm{p}=0.5027$. Very significant decrease of the mean land maze time between study group B (E) and study group C (F) - p=0.0002.

weeks follow-up; but, they were similar to normal (control) rats after 16 weeks follow-up.

At the end of the investigation period the animal of the study group underwent euthanasia, and the anterior portion of the neck were surgically open for the assessment of the ligated carotids. In both sides fibrous cord were seen in these arterial vessels. Additionally, thin arterial branches could be seen running in the carotid fibrous cord and in thigh attachment to the trachea (Figures 7 and 8).

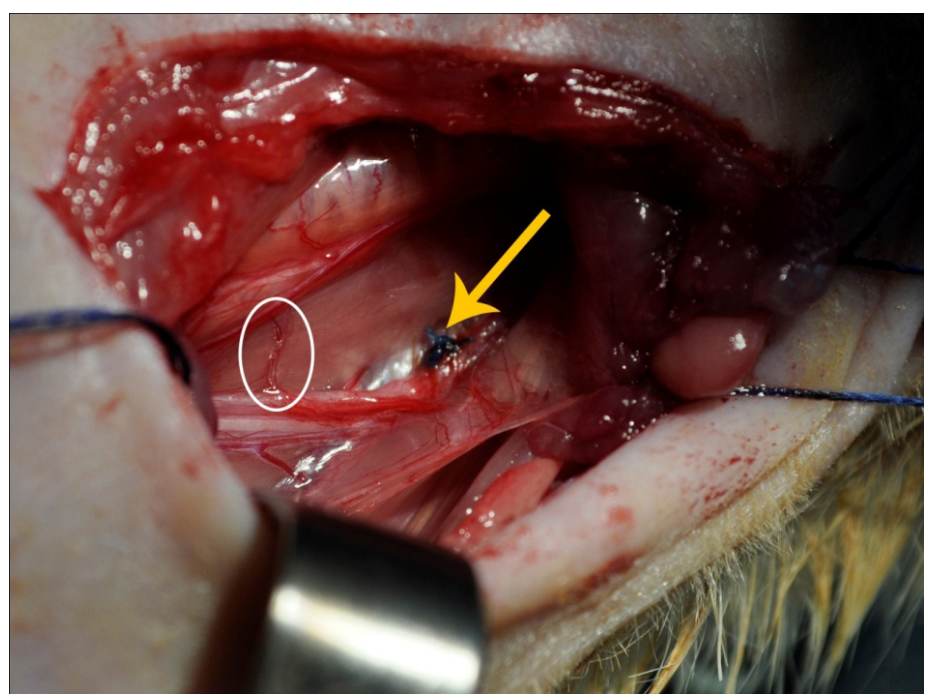

FIGURE 7 - Adaptive arteriogenesis after BCCA. Yellow arrow indicating the ligature of the left carotid artery. White circle and blue arrow indicating one of the thin colateral artery close by to trachea. 


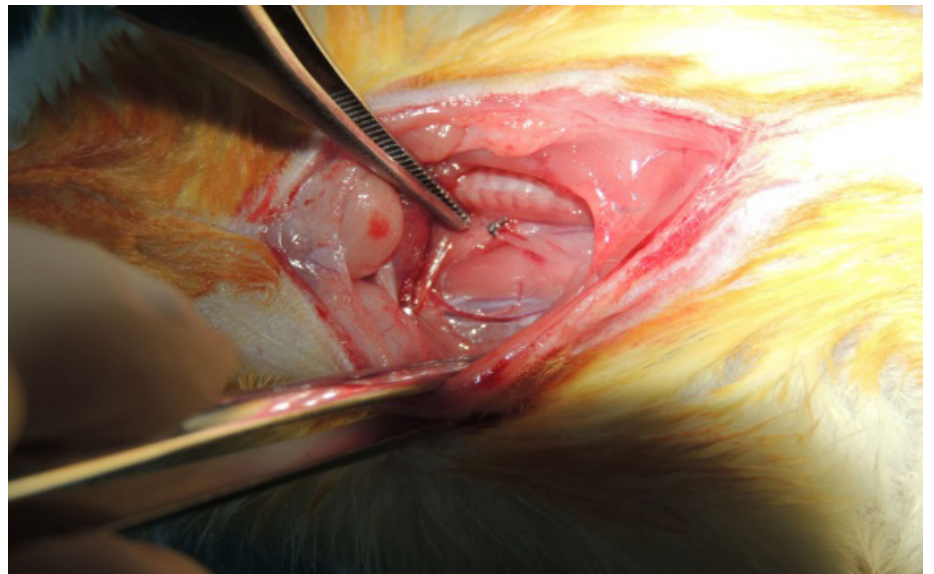

FIGURE 8 - Fibrous cord resulting from left BCCA. Thin arteries (arrows) running attached to the fibrous cord and posterior aspect of this cord.

\section{Discussion}

The majority of papers dealing with chronic encephalic hypoxemia due to bilateral common carotid ligation (BCCL) indicate that it is associated with neuron apoptosis, mainly in the hippocampus, cerebral cortex, and caudate affecting the microenvironment vital for the central nervous system functions ${ }^{3,5,13-24}$, producing cognitive changes measured by radial maze tests. These changes are supposedly reverted using several therapeutically interventions ${ }^{13-24}$.

A chronic, sustained reduction of cerebral blood flow can cause neuropath logical changes that may lead to various neurological deficits such as chronic ischemic infarction or vascular dementia (VD) via the secondary impairment of cerebral glucose metabolism and chronic energy production ${ }^{25}$. However, one has to realize that the vascular impairment of the arterial supply for the brain is different when comparing human being and rats. As the BCCA is performed in rats there is an almost immediately compensation from the collaterals arterial branches from the vertebral arteries.

In murine animals, apart from neuron plasticity, there is a proved adaptive mechanism of encephalic improvement after global ischemic damage (neurogenesis) particularly in the hippocampus area ${ }^{26,27}$.

Effects on adaptive cerebral arteriogenesis has been demonstrate in other model of cerebral hypo perfusion, so called three vessel occlusion (bilateral vertebral plus unilateral common carotid artery) occlusion $(3-\mathrm{VO})^{28,29}$. It is likely that the same phenomenon occurs after BCCL in rats.

The findings in the present investigation lend support to the hypothesis that, in rats, there is a natural adaptive mechanisms (hemodynamic, neuronal plasticity, neurogenesis and arteriogenesis), which prevent these animals from dying, and as the post-operative time goes by there is a non-interventional restoration of the rat brain blood perfusion neuron encephalic population, and consequently the natural improvement of the neurocognitive functions. The evidence from this study is that the conclusions from previous investigations ${ }^{13-24}$ performed in 2012; as well as in the years before may be not useful for translational human being purpose. As a consequence we suggest that there is a need for searching an alternative animal model, rather than BCCL in rats, which can mimic chronic cerebral hypo perfusion and could allow for therapeutically approaches aiming at to prevent or to improve the neurocognitive deficit produced after chronic decreasing the arterial supply to the encephalon.

\section{Conclusions}

From the data one can assume that the improvement of the neurocognitive deficits after 16 weeks post-operative followup of rats that underwent BCCL is a natural adaptive phenomenon. Furthermore, the interpretation of these findings is that is not realistic to allow translational information from this animal model for therapeutically approaches aiming at to prevent, or to improve brain damage in human beings suffering from chronic deprivation of adequate blood supply.

\section{References}

1. Plum F, Posner JB, Alvord Jar EC. Edema and necrosis in experimental cerebral infarction. Arch Neurol. 1963;9:563-70.

2. Payan H M, Leveine S, Strevel R. Effects of cerebral ischemia in various strains of rats. Proc Soc Exp Biol Med. 1965;120:208-9.

3. Farkas E, Luiten PG, Bari F. Permanent, bilateral common carotid artery occlusion in the rat: a model for chronic cerebral hypoperfusion-related neurodegenerative diseases. Brain Res Rev. 2007;54:162-80.

4. Kunimatsu T, Asai S, Kanematsu K, Kohno T, Misaki T, Ishikawa $\mathrm{K}$. Effects of glutamate receptor agonist on extracellular glutamate dynamics during moderate cerebral ischemia. Brain Res. 2001;923:178-86.

5. Otori T, Katsumata T, Katayama Y, Terashi A. Measurement of regional cerebral blood flow and glucose utilization in rat brain under chronic hypoperfusion conditions following bilateral carotid artery occlusion. Analyzed by autoradiographical methods. Nippon IkaDaigakuZasshi. 1997;64:428-39.

6. Farooqui AA, Horrocks LA. Excitatory amino acid receptors, neural membrane phospholipid metabolism and neurological disorders. Brain Res Brain Res Rev. 1991;16:171-91.

7. Clark JD, Schievella AR, Nalefski EA, Lin LL. Cytosolic phospholipase A2. J Lipid Mediat Cell Signal. 1995;12:83-17.

8. Dennis EA. Diversity of group types, regulation, and function of phospholipase A2. J Biol Chem. 1994;269:13057-60.

Li RC, Row BW, Gozal E, Kheirandish L, Fan Q, Brittian KR, Guo SZ, Sachleben LR Jr, Gozal D. Cyclooxygenase 2 and intermittent hypoxia-induced spatial deficits in the rat. Am J Respir Crit Care Med. 2003;168:469-75. 
Bolanos JP, Almeida A. Roles of nitric oxide in brain hypoxia-ischemia. Biochim Biophys Acta. 1999;1411:415-36.

Rodrigo J, Fernandez AP, Serrano J, Peinado MA, Martinez A. The role of free radicals in cerebral hypoxia and ischemia. Free Radic Biol Med. 2005;39:26-50.

Irikura K, Morii S, Miyasaka Y, Yamada M, Tokiwa K, Yada K. Impaired autoregulation in an experimental model of chronic cerebral hypoperfusion in rats. Stroke. 1996;27:1399-404.

Kwak PA, Lim SC, Ham SR, Shon YM, Kim Yi. Supra-additive neuroprotection by renexin, a mixed compound of ginkgo biloba extract and cilostazol, against apoptotic white matter changes in rat after chronic cerebral hypoperfusion. J Clin Neurol. 2012;8(4):284-92.

Won JS, Kim J, Annamalai B, Shunmugavel A, Singh I, Singh AK. Protective role of S-nitrosoglutathione (GSNO) against cognitive impairment in rat model of chronic cerebral hypoperfusion. $\mathrm{J}$ Alzheimers Dis. 2013;34(3):621-35.

Feng Z, Lu Y, Wu X, Zhao P, Li J, Peng B, Qian Z, Zhu L. Ligustilide alleviates brain damage and improves cognitive function in rats of chronic cerebral hypoperfusion. J Ethnopharmacol. 2012;144(2):313-21.

Liu YH, Li SW, Zheng QL. Effects of bushenjiannao recipe on the content of acetylocholine and the hipppcampal ERK1 and ERK2 protein expressions of vascular dementia rats. Zhongguo Zhong Xi Yi Jie He Za Zhi. 2012;32(4):504-9.

Wang YH, He XL, Li XX, Qin HL, Du GH. Effects of the effective component group of Chinese herbal medicine Xiaoxuming Decoction on brain mitochondria in rats with chronic cerebral ischemia.Zhong Xi Yi Jie He Xue Bao. 2012;10(5):569-76.

Wei W, Zhang W, Huang Y, Li Y, Zhu G, Chen F, Li J. The therapeutic effect of (DL)-3-n-butylphthalide in rats with chronic cerebral hypoperfusion through downregulation of amyloid precursor protein and matrix metalloproteinase-2. J Int Med Res. 2012;40(3):967-75.

Luo Y, Zhao HP, Zhang J, Wang J, Yang WL, Yang M, Liao ZG. Effect of ferulic acid on learning and memory impairments of vascular dementia rats and its mechanism of action. Yao Xue Xue Bao. 2012;47(2):256-60.

Zhang HN, Wu J, Jin T, Chang M, Sun L. Transient elevation of synaptosomalmitoenergetic proteins and Hsp70 early in a rat model of chronic cerebrovascular hypoperfusion. Neurol Sci. 2013;34(4):471-7.

Gong X, Ma M, Fan X, Li M, Liu Q, Liu X, Xu G. Down-regulation of IGF-1/IGF-1R in hippocampus of rats with vascular dementia. Neurosci Lett. 2012;28;513(1):20-4.
Cechetti F, Worm PV, Lovatel G, Moysés F, Siqueira IR, Netto CA. Environmental enrichment prevents behavioral deficits and oxidative stress caused by chronic cerebral hypoperfusion in the rat. Life Sci. 2012;91(1-2):29-36.

Zhang YG, Xiong KR. Effects of electroacupuncture combined with compound SalviaeMiltiorrhizae tablet on the expressions of brain derived neurotrophic factor and vascular endothelial growth factor in hippocampus CA1 of chronic cerebral ischemia rats. ZhongguoZhong Xi Yi Jie He ZaZhi. 2012;32(5):643-6.

Quartu M, Serra MP, Boi M, Pillolla G, Melis T, Poddighe L, Del Fiacco M, Falconieri D, Carta G, Murru E, Cordeddu L, Piras A, Collu M, Banni S. Effect of acute administration of Pistacialentiscus L. Essential oil on rat cerebral cortex following transient bilateral common carotid artery occlusion. Lipids Health Dis. 2012;11:8.

Beal MF, Hyman BT, Koroshetz W. Do defects in mitochondrial energy metabolism underlie the pathology of neurodegenerative diseases? Trends Neurosci. 1993;16:125-31.

Liu J, Solway K, Messing RO, Sharp FR. Increased neurogenesis in the dentate gyrus after transient global ischemia in Gerbils. J. Neurosci. 1998;18:7768-78.

Yao-Fang T, Preston E, Wojtowicz JM. Enhanced post-ischemic neurogenesis in aging rats. Front Neurosci. 2010;4(163):1-6.

Busch HJ, Buschmann IR, Mies G, Bode C, Hossmann KA. Arteriogenesis in hypoperfused rat brain. J Cereb Blood Flow Metab. 2003;23:621-8.

Duelsner A, Gatzke N, Glaser J, Hillmeister P, Li M, Lee EJ, Lehmann K, Urban D, Meyborg H, Stawowy P, Busjahn A, Nagorka S, Persson AB, Buschmann IR. Acetylsalicylic acid, but not clopidogrel, inhibits therapeutically induced cerebral arteriogenesisin the hypoperfused rat brain. J Cereb Blood Flow Metab. 2012;32(1):105-14.

\section{Correspondence:}

Carlos Teixeira Brandt

Avenida Boa Viagem, 5030/1302

51011-000 Recife - PE Brasil

Tel.: (55 81)3342-0830

carlosbrandt@bol.com.br

Received: Sept 10, 2013

Review: Nov 12, 2013

Accepted: Dec 16, 2013

Conflict of interest: none

Financial source: Medical Sciences, Faculty of Campina Grande

${ }^{1}$ Research performed at Experimental Research Unit, Campina Grande Faculty of Medicine (FCM), Campina Grande-PB, Brazil. Part of PhD degree thesis, Postgraduate Program in Surgery, Federal University of Pernambuco (UFPE). Tutor: Prof. Dr. Carlos Teixeira Brandt. 